03,06

\title{
Влияние частоты переменного электрического поля на температурные спектры импеданса керамического мультиферроика $\mathrm{LuFe}_{2} \mathrm{O}_{4}$
}

\author{
(C) Р.А. Алиев ${ }^{1}$, А.Г. Гамзатов ${ }^{1}$, Г.М. Гаджиев ${ }^{1,}$, Н.С. Абакарова ${ }^{1}$, А.Р. Кауль ${ }^{2}$, \\ М. Маркелова ${ }^{2}$ Л.Л. Эмирасланова ${ }^{3}$ \\ ${ }^{1}$ Институт фризики ДагНЦ РАН, \\ Махачкала, Россия \\ ${ }^{2}$ Московский государственный университет, \\ Москва, Россия \\ ${ }^{3}$ Дагестанский государственный университет, \\ Махачкала, Россия \\ ฯ E-mail: Hadzhygm@mail.ru
}

Измерены температурные спектры импеданса керамического мультиферроика $\mathrm{LuFe}_{2} \mathrm{O}_{4}$ в интервале температур 100-400 К при воздействии измерительного синусоидального напряжения с частотой $20 \mathrm{~Hz}-120 \mathrm{MHz}$. Для частот измерительного сигнала в области $30-70 \mathrm{MHz}$ на спектрах наблюдаются особенности в виде аномального положительного температурного коэффициента сопротивления в диапазоне температур $200-260$ К. Данные особенности интерпретированы на основе представлений об обобщенной барьерной модели.

Работа выполнена в рамках государственного задания ФАНО России (тема № 0203-2016-0005).

DOI: 10.21883/FTT.2018.06.45977.06M

Поиск функциональных материалов, электрическими свойствами которых можно управлять с помощью магнитного поля, а магнитными свойствами - посредством электрического поля, вызвал среди исследователей бурный интерес к изучению мультиферроиков [1]. Мультиферроики - материалы, сочетающие в себе одновременно магнитное и электрическое упорядочения, между которыми имеется эффект взаимовлияния (магнитоэлектрический эффект) [2]. Одним из таких материалов является соединение феррит лютеция $\mathrm{LuFe}_{2} \mathrm{O}_{4}$. Этот материал имеет гексагональную слоистую структуру с пространственной группой $R \overline{3} m$. В такой структуре имеются двойные слои треугольных решеток атомов железа $(W$-слои $)$, которые содержат одинаковое число $\mathrm{Fe}^{+2}$ и $\mathrm{Fe}^{+3}$ ионов [3]. Возникновение электрической поляризации в данных материалах обусловлено процессами электронной корреляции и геометрической фрустрацией $\left(\mathrm{Fe}^{2+} / \mathrm{Fe}^{3+}\right)$ зарядов в области температур между $350 \mathrm{~K}$ и $500 \mathrm{~K}$, приводящей к $2 \mathrm{D}$ зарядовому упорядочению, а при температуре ниже $320 \mathrm{~K}$ - к 3D зарядовому упорядочению. Возникающее в результате такого процесса 3D зарядовое упорядочение, в свою очередь, индуцирует магнитное упорядочение при уменьшении температуры ниже $250 \mathrm{~K}[4,5]$.

Близость температуры зарядового и магнитного упорядочения к комнатной делает мультиферроик $\mathrm{LuFe}_{2} \mathrm{O}_{4}$ привлекательным материалом для практического применения в качестве элементов энергонезависимой памяти, считывающих магнитных головок, элементов сенсорики [6-8]. Соединения $\mathrm{LuFe}_{2} \mathrm{O}_{4}$ характеризуются высоким значением отрицательного температурного коэффициента сопротивления (ТКС) в области температур 150-320 K, что указывает на возможность их использования в различных терморегулирующих устройствах. В большинстве работ, посвященных импедансной спектроскопии соединений $\mathrm{LuFe}_{2} \mathrm{O}_{4}$, измерения, в основном, были выполнены при частоте тестирующего переменного электрического поля от $20 \mathrm{~Hz}$ до $1 \mathrm{MHz}$ [7-12]. Практически отсутствуют работы, в которых обсуждается аномальное поведение ТКС $\mathrm{LuFe}_{2} \mathrm{O}_{4}$ в области температуры магнитного упорядочения $\sim 250 \mathrm{~K}$ при частотах измерительного сигнала несколько десятков МHz. В имеющихся единичных работах [13] слабо наблюдаемые особенности электропроводности для частот больше $1 \mathrm{MHz}$ вблизи температуры магнитного фазового перехода не анализируются. В данной работе мы сообщаем об аномальном поведении ТКС керамического $\mathrm{LuFe}_{2} \mathrm{O}_{4}$ вблизи температуры $\sim 250 \mathrm{~K}$ при частотах тестирующего электрического сигнала в диапазоне 30-70 MHz.

В нашей работе использовались керамические образцы $\mathrm{LuFe}_{2} \mathrm{O}_{4}$ из той же технологической серии, что и образцы, исследованные в [5]. Образцы представляли собой дискообразные таблетки диаметром $\sim 6 \mathrm{~mm}$ и толщиной около $1.5 \mathrm{~mm}$. На противоположные грани круглого сечения методом магнетронного распыления были нанесены серебряные контакты с диаметром $2.9 \mathrm{~mm}$. Измерительные провода припаивались к контактам индиевым припоем. Температурные зависимости импеданса $Z$ и угла сдвига фазы между током и напряжением $\theta$ переменного тестирующего сигнала одновременно измерялись на высокочастотном LCR-метре WK-6500P. Охлаждение образца осуществлялось в заливном азот- 


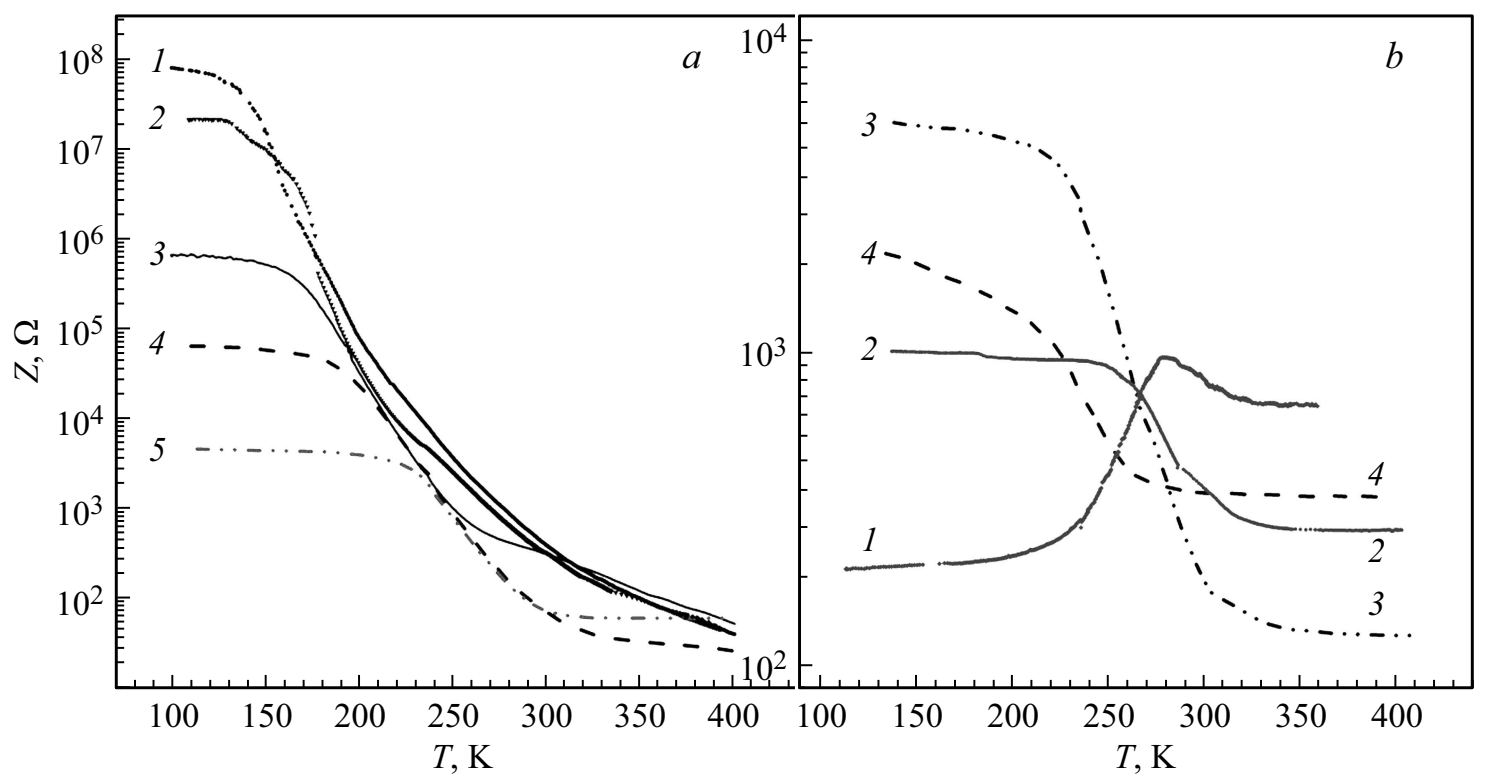

Рис. 1. Температурные спектры импеданса $\mathrm{LuFe}_{2} \mathrm{O}_{4}:$ a) $1-20 \mathrm{~Hz}, 2-5 \mathrm{kHz}, 3-50 \mathrm{kHz}, 4-500 \mathrm{kHz}, 5-5 \mathrm{MHz}$. b) $1-50 \mathrm{MHz}, 2-80 \mathrm{MHz}, 3-100 \mathrm{MHz}, 4-120 \mathrm{MHz}$.

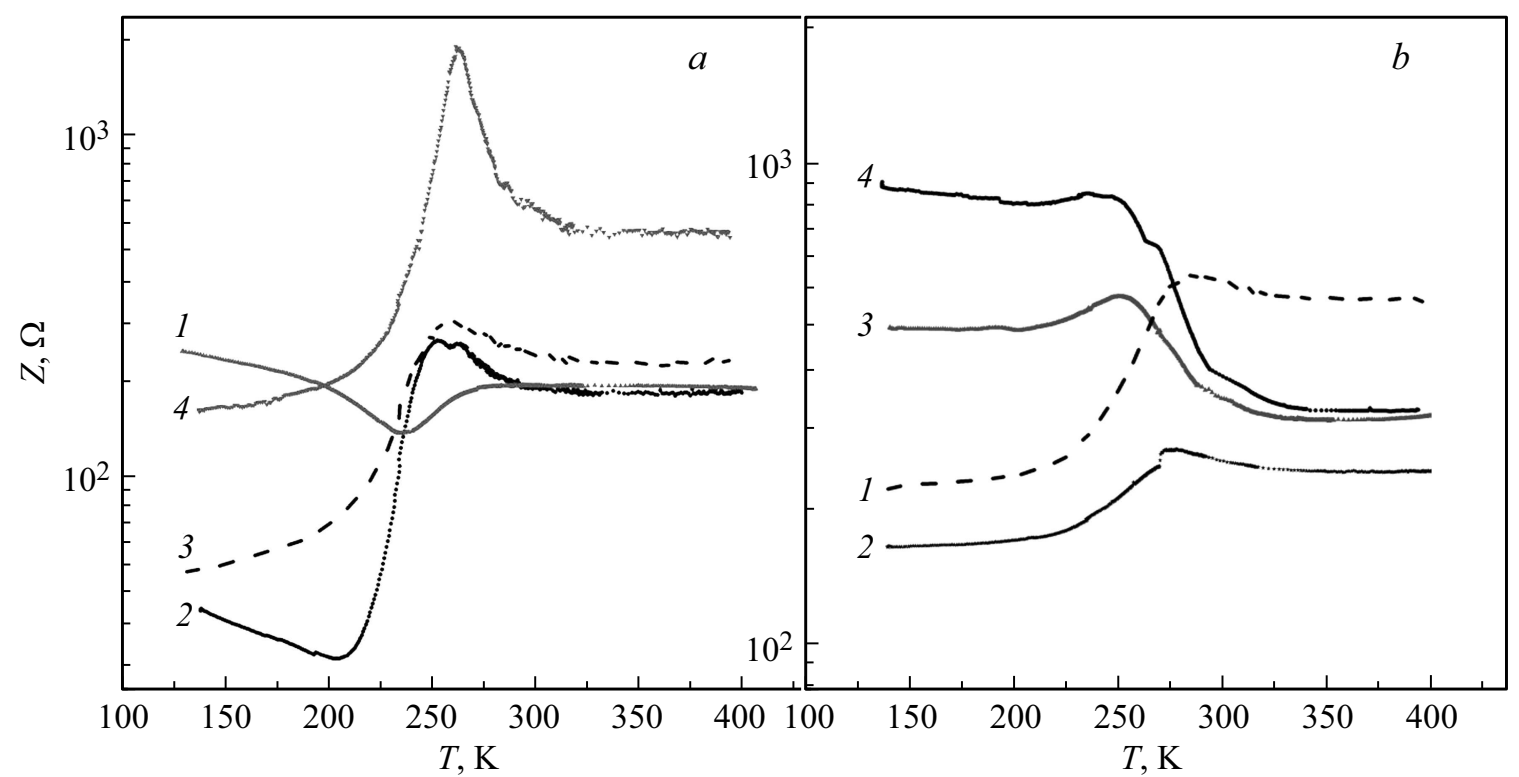

Рис. 2. Температурная зависимость импеданса в области частот $30-70 \mathrm{MHz}$. a) $1-30 \mathrm{MHz}, 2-35 \mathrm{MHz}, 3-40 \mathrm{MHz}$, $4-45 \mathrm{MHz}$. b) $1-55 \mathrm{MHz}, 2-60 \mathrm{MHz}, 3-65 \mathrm{MHz}, 4-70 \mathrm{MHz}$.

ном криостате. Измеренные $Z$ и $\theta$ пересчитывались в действительную $Z^{\prime}$ и мнимую $Z^{\prime \prime}$ компоненты импеданса. Температура образца контролировалась с помощью калиброванной медь-константановой термопары, один спай которой помещался в сосуд Дьюара с тающим льдом. Ошибка измерения температуры в области $100-350 \mathrm{~K}$ не превышала $1 \mathrm{~K}$. Скорость нагрева образца поддерживалась равной $2 \mathrm{~K} / \mathrm{min}$. Амплитуда тестирующего переменного напряжения составляла $0.5 \mathrm{~V}$.

На рис. $1, a, b$ представлены температурные спектры импеданса (ТСИ) для различных частот измерительного переменного электрического поля. Все спектры, за исключением измеренного на частоте $50 \mathrm{MHz}$, характеризуются отрицательным ТКС и претерпевают излом наклона в области от $\sim 300 \mathrm{~K}$ до $\sim 320 \mathrm{~K}$. То есть зависимость сопротивления от температуры носит полупроводниковый характер, как и в предыдущих работах [11], а частотная зависимость $Z$, в целом, описывается в приближении дебаевской релаксации. Из рис. 1 видно, что ТСИ для $50 \mathrm{MHz}$ имеет зеркально-симметричный характер по отношению к другим спектрам. Для более тщательной проверки данного эффекта мы измерили ТСИ в области частот тестирующего сигнала от $30 \mathrm{MHz}$ до $70 \mathrm{MHz}$. 
На рис. 2, $a, b$ приведены результаты измерений ТСИ для частот тестирующего сигнала в указанной выше области. Из рисунка видно, что при изменении частоты переменного поля, изменяется соотношение низкотемпературной $(T<255 \mathrm{~K})$ и высокотемпературной $(T>255 \mathrm{~K})$ ветвей ТСИ. ТСИ для частот $35 \mathrm{MHz}$, $40 \mathrm{MHz}, 45 \mathrm{MHz}, 55 \mathrm{MHz}$ и $65 \mathrm{MHz}$ характеризуются большими значениями положительного ТКС в области температур от $200 \mathrm{~K}$ до $\sim 255 \mathrm{~K}$, после которой ТКС меняет знак. ТСИ для $45 \mathrm{MHz}$ обладает ярко выраженным максимумом при $T=262 \mathrm{~K}$ с наибольшим значением $Z$ в максимуме. Рис. 2,b показывает, что с увеличением частоты переменного измерительного напряжения низкотемпературная ветвь в спектре поднимается и в ТСИ для $70 \mathrm{MHz}$ она располагается выше высокотемпературной ветви. При дальнейшем увеличении частоты спектры вновь приобретают вид с отрицательным ТКС, как на рис. 1. Рис. 3 демонстрирует частотную эволюцию $(30-80 \mathrm{MHz})$ модуля импеданса $Z$, которая визуализирует соотношение импедансов низкотемпературной и высокотемпературной областей ТСИ при трех фиксированных температурах. Точки на рис. 3 соединены огибающими линиями.

Как известно [14], положительное значение угла сдвига фазы $\theta$ между током и напряжением в цепи переменного тока обусловлено наличием элемента $(L)$ индуктивности $(\theta \sim \operatorname{arctg}(L))$ и соответственно отрицательное значение угла указывает на $(C)$ емкостный характер импеданса $(-\theta \sim \operatorname{arctg}(C))$ в зависимости от вида соединения реактивных элементов в цепи). В области температур от $200 \mathrm{~K}$ до $\sim 255 \mathrm{~K}$ для частот 35 , 40, 45 и $50 \mathrm{MHz}$, ТСИ которых имеют наиболее характерный вид, углы сдвига фазы демонстрируют положительные значения (рис. 4). При этом наблюдается корреляция между ростом положительных значений $\theta$

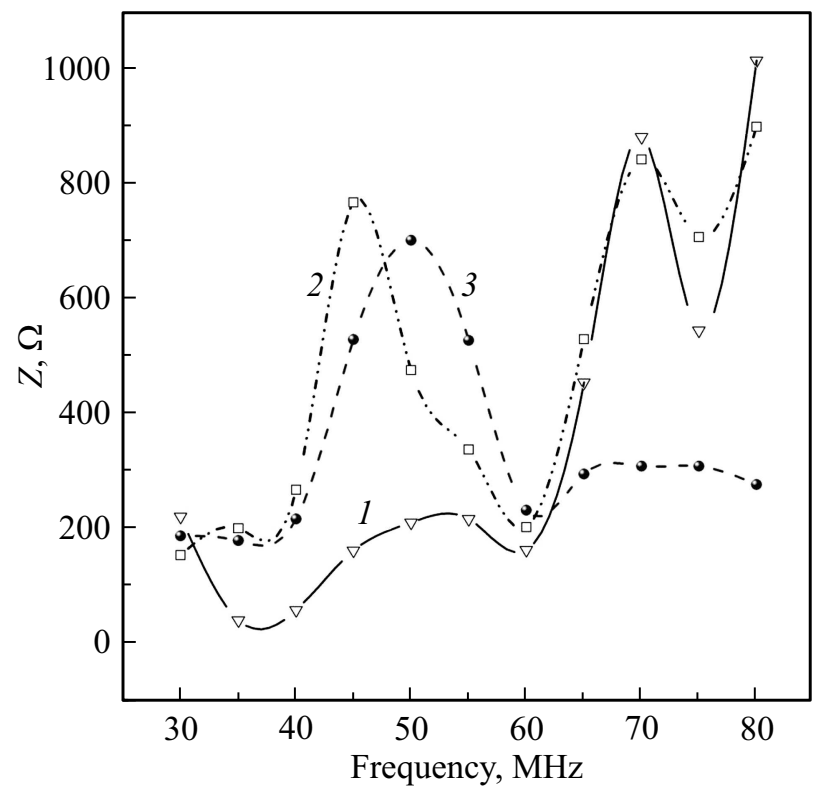

Рис. 3. Зависимость $Z$ от частоты измерительного напряжения при температуре: $1-150 \mathrm{~K} ; 2-250 \mathrm{~K} ; 3-350 \mathrm{~K}$.

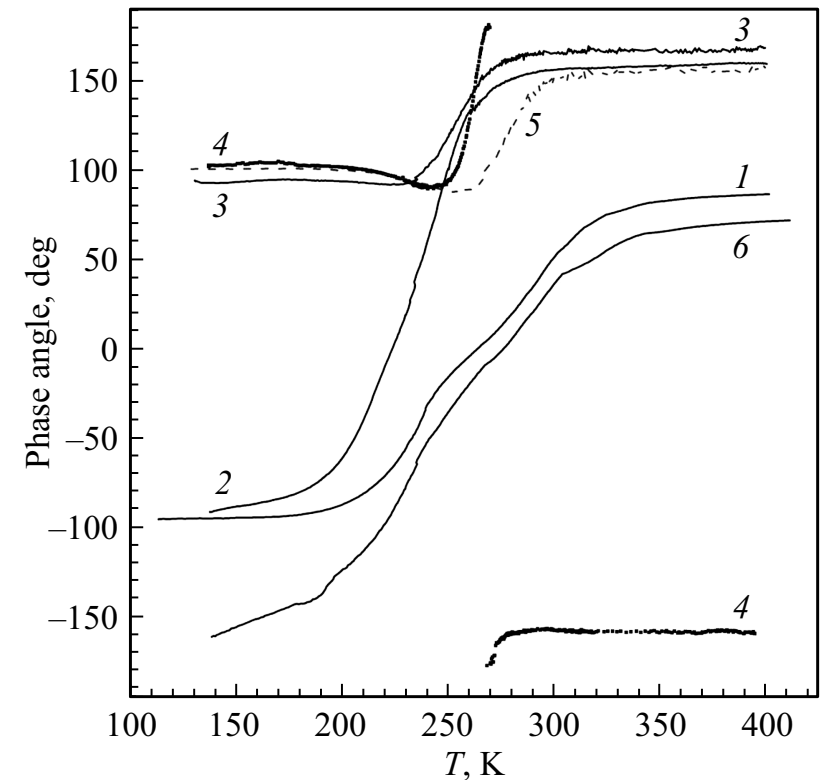

Рис. 4. Зависимости $\theta(T)$, измеренные на частоте переменного напряжения: $1-5 \mathrm{MHz}, 2-35 \mathrm{MHz}, 3-40 \mathrm{MHz}$, $4-45 \mathrm{MHz}, 5-50 \mathrm{MHz}, 6-100 \mathrm{MHz}$.

в интервале температур от $\sim 220 \mathrm{~K}$ до $\sim 260 \mathrm{~K}$ (кривые $2,3,4,5$ рис. 4$)$ и ростом $Z$ в соответствующих ТСИ (рис. 2). Резкий рост значения $Z$ в ТСИ для $35 \mathrm{MHz}$, $40 \mathrm{MHz}, 45 \mathrm{MHz}$ и $55 \mathrm{MHz}$ при увеличении температуры до $\sim 260 \mathrm{~K}$ (рис. 2) мы связываем с проявлением индуктивной составляющей полного сопротивления, т.е. с появлением у образца индуктивных свойств. Широкие пики в области $40-60 \mathrm{MHz}$ на рис. 3 (кривые 2,3) указывают на наличие в цепи параллельной цепочки из элемента индуктивости $L$ и емкости $C$, последовательно включенной с активным сопротивлением $R$ [15].

За появление импеданса индуктивного типа могут быть ответственны несколько независимых механизмов: инерционно-релаксационный характер движения доменных (электрическая поляризация) стенок [12]; релаксационный механизм формирования обедненного слоя (барьера Шоттки) на границе зерен или на контакте электрод-образец. Согласно представлениям обобщенной барьерной модели в поликристаллических окисных полупроводниках типа ферритов локальные барьеры могут быть на границах зерен (в виде высокоомных прослоек и запирающих слоев), на неоднородностях типа дислокаций, магнитных (и электрических) доменов и т. Д. [16].

Электрические свойства керамики, состоящей из зерен с полупроводниковыми свойствами с сопротивлением $\rho_{g}$, окруженных изолирующими слоями с сопротивлением $\rho_{b} \gg \rho_{g}$, можно описать соотношением типа формулы Дебая [17]:

$$
\rho=\rho_{g}+X \frac{\rho_{b}}{1+\omega^{2} \tau^{2}},
$$

где $X$ - отношение толщины барьера к толщине зерна; $\omega=2 \pi f-$ циклическая частота электрического поля; 


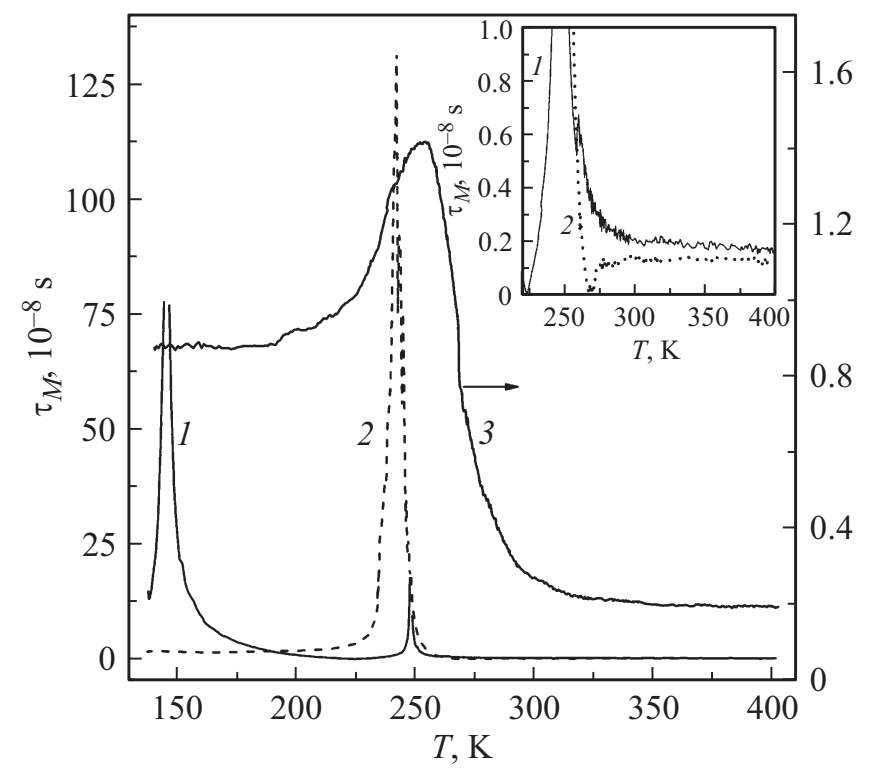

Рис. 5. Температурная зависимость максвелловского времени релаксации, рассчитанная для частоты измерительного сигнала: $1-35 \mathrm{MHz}, 2-45 \mathrm{MHz}, 3-55 \mathrm{MHz}$. На вставке увеличенный фрагмент зависимости $\tau_{M}(T)$ для кривых 1,2 .

$\tau$ - время диэлектрической релаксации. Для высокоомных полупроводников, какими являются ферриты, можно считать $\tau \approx \tau_{M}, \tau_{M}-$ максвелловское время релаксации барьерного слоя.

На рис. 5 представлены температурные зависимости $\tau_{M}$ для трех частот синусоидального измерительного напряжения, на которых наблюдаются наиболее типичные ТСИ с положительным ТКС (рис. 2, $a$, кривые 2,4; рис. 2, $b$, кривая 1). Максвелловское время рассчитывалось по формуле [16] $\tau_{M}=\frac{\varepsilon^{\prime}}{\varepsilon^{\prime \prime}} \frac{1}{\omega}$, где $\varepsilon^{\prime}$, $\varepsilon^{\prime \prime}$ - действительная и мнимая части комплексной диэлектрической проницаемости, которые для каждого конкретного значения температуры рассчитывались по формулам [15]: $\varepsilon^{\prime}=Y^{\prime \prime} / \omega \cdot C_{0} ; \varepsilon^{\prime \prime}=Y^{\prime} / \omega \cdot C_{0}$, где $Y^{\prime}=\cos \theta / Z$ и $Y^{\prime \prime}=\sin \theta / Z-$ реальная и мнимая компоненты полной проводимости $Y, C_{0}$ - геометрическая емкость контактных электродов в вакууме.

Из (1) следует, что при $\omega \tau \ll 1$ изменение электросопротивления, в основном, определяется параметром $X$, а при $\omega \tau \gg 1-$ в большей степени объемными свойствами зерна. При подаче обратного напряжения измерительного сигнала на барьерную структуру, в течение времени $1 / 4 f$ происходит формирование запирающего слоя, во вторую четверть периода поле уменьшается, а затем оно меняет знак и механизм повторяется для противоположной границы зерна. Когда $1 / 4 f>\tau_{M}$ логично предположить, что параметр $X$ будет максимальным, но нельзя не учитывать влияние глубоких центров (роль которых могут играть различные структурные дефекты в зернах и на их границах) на толщину запирающего слоя [18]. После подачи обратного напряжения тестового сигнала с периодом $1 / f>\tau_{M}$ происходит уменьшение толщины запирающего слоя (по сравнению с начальной величиной при $\left.t=\tau_{M}\right)$ в течение времени релаксации заполнения глубокого уровня, которое обычно больше $\tau_{M}$ [18]. Для случая $1 / 4 f<\tau_{M}$, измерительная аппаратура регистрирует значение $Z$ с $X<X_{\max }$. Следовательно, при $\omega \tau \ll 1$ и $\omega \tau \gg 1$ параметр $X$ из (1) не достигает максимального значения. Максимальное значение $X$, как следует из вышеприведенных рассуждений, будет достигнуто при $1 / 4 f \approx \tau_{M}$, т. е. при возникновении резонансного состояния $(\omega \tau \approx 1)$.

Рассчитанные значения $\tau_{M}$ при температуре $T=T_{\max }$ для ТСИ, измеренных на частототах $35 \mathrm{MHz}, 45 \mathrm{MHz}$ и $55 \mathrm{MHz}$ (рис. 2) составили: $0.98 \cdot 10^{-8} \mathrm{~s}, 0.53 \cdot 10^{-8} \mathrm{~s}$ и $0.33 \cdot 10^{-8} \mathrm{~s}$ соответственно, а $1 / 4 f$ для этих частот равны $0.7 \cdot 10^{-8} \mathrm{~s}, 0.55 \cdot 10^{-8} \mathrm{~s}$ и $0.45 \cdot 10^{-8} \mathrm{~s}$ соответственно. $T_{\max }$ для данных ТСИ (рис. 2) равны 254, 263 и $289 \mathrm{~K}$ соответственно. Как видно из рис. 2 , наиболее ярко выраженный ТСИ с положительным ТКС имеет место при частоте $45 \mathrm{MHz}$, то есть, когда $1 / 4 f \approx \tau_{M}$.

По нашему мнению, с учетом вышесказанного, перестройка спектров с отрицательным ТКС в спектры с положительным ТКС происходит при увеличении сопротивления барьерного слоя на границе зерна с приближением $1 / 4 f$ периода измерительного переменного напряжения к $\tau_{M}$ и достигает максимального значения при $1 / 4 f \approx \tau_{M}$. При дальнейшем уменьшении периода сигнала (увеличении $f$ ) измерительная аппаратура не успевает регистрировать граничные эффекты зерна и проводимость определяется его объемными свойствами, т.е. в этом случае проводимость является прыжковой, которая зависит от частоты по степенному виду.

Важно отметить, что как увеличение ширины обедненной области барьера Шоттки, так и ее уменьшение в течение периода измерительного сигнала вызывает модуляцию проводимости. Такая модуляция сопровождается появлением у образца индуктивных свойств, которые, по всей видимости, проявляются максимально при резонансных условиях $(\omega \tau \approx 1)$. При фиксированной частоте тестового сигнала, резонансное состояние может быть достигнуто при изменении $L$ или $C$ образца, что, в нашем случае, достигается посредством изменения температуры образца.

С практической точки зрения, наблюдаемые в работе аномальные положительные ТКС могут быть использованы в устройствах управления температурой и ограничения тока. Поскольку особенности в ТСИ проявляются в окрестности температуры магнитного фазового перехода, то необходимы дальнейшие исследования этих соединений в постоянном магнитном и электрическом поле. Такие исследования могли бы выявить природу влияния доменной конфигурации на описанные выше эффекты.

\section{Список литературы}

[1] J.F. Scott. Science 315, 954 (2007).

[2] N.A. Hill. J. Phys. Chem. B 104, 6694 (2000).

[3] N. Ikeda, H. Ohsumi, K. Ohwada, K. Ishi, T. Inami, K. Kakurai, Y. Murakamim K. Yoshii, S. Mori, Y. Horibe, H. Kito. Nature 436, 1136 (2005). 
[4] S.V. Sheong, M. Mostovoy. Nature Mater. 6, 13 (2007).

[5] Y.B. Kudasov, D.A. Maslov. Phys. Rev. B 86, 214427 (2012)

[6] M. Bibes, A. Barthelemy. IEEE Trans. Electron Devices 54, 1003 (2007)

[7] J. Van den Brink, D.I. Khomskii. J. Phys. Condens. Matter 20, 434217 (2008)

[8] A.P. Pyatakov, A.K. Zvezdin. Phys. Usp. 182, 593 (2012).

[9] D.S.F. Viana, R.A.M. Gotardo, L.F. Cotica, M. Ozon-Dionysio, D. Garcia, T.A. Eiras. A.A. Coelho. J. Appl. Phys. 110, 034108 (2011).

[10] Ying Hou, Yiping Yao, Sining Dong, Xi Huang, Xuefeng Sun, Xiaoguang Li. J. Mater. Res. 27, 6, 922 (2012).

[11] S. Lafuerza, J. Garcia, G. Subias, T. Blasco, K. Conder, E. Pomjakushina. Phys. Rev. B 88, 085130 (2013).

[12] Yu.B. Kudasov, M. Markelova, D.A. Maslov, V.V. Platonov, O.M. Surdin, A. Kaul. Phys. lett. A 380, 3932 (2016)

[13] D. Nierman, F. Waschkowski, J. de Groot, M. Angst, J. Hemberger. Phys. Rev. Lett. 109, 016405 (2012).

[14] Л.А. Бессонов. Теоретические основы электротехники. Высш. шк., М. (1996). 640 с.

[15] Н.А. Поклонский, Н.И. Горбачук. Основы импедансной спектроскопии композитов. Изд. центр Белорус. гос. унта, Минск (2005). 132 с.

[16] П.Т. Орешкин. Физика полупроводников и диэлектриков. Высш. шк., М. (1977). 448 с.

[17] М. Лайнс, А. Гласс. Сегнетоэлектрики и родственные им материалы. Мир, М. (1981). С. 594

[18] Л.С. Берман, А.А. Лебедев. Емкостная спектроскопия глубоких центров в полупроводниках. Наука, Л. (1981). C. 28 . 